\section{Waveguide-type optical passive ring resonator gyro using phase modulation spectroscopy technique}

\author{
Huilian Ma, Xulin Zhang, Zhonghe Jin, and Chun Ding \\ Zhejiang University, Department of Information \\ Science \& Electronic Engineering, Hangzhou 310027 \\ China \\ E-mail: mahl@zju.edu.cn
}

\begin{abstract}
We report the first demonstration of silica waveguide optical passive ring resonator gyro (OPRG) based on the phase modulation spectroscopy technique. The ring resonator is composed of a 6 -cm-long silica waveguide. Observed from the resonance curve, the free spectral range (FSR) of the resonator, the full width at half maximum (FWHM) of the resonance curve, the finesse $(F)$ of the resonator, and the resonance depth are $3.4 \mathrm{GHz}, 62 \mathrm{MHz}, 54.8$, and $70 \%$, respectively. The detection sensitivity of this OPRG will be $7.3 \times 10^{-5} \mathrm{rad} / \mathrm{s}$. In the experiments, there is an acoustic-optical modulator (AOM) in each light loop. We lock the lasing frequency at the resonance frequency of the silica waveguide ring resonator for counterclockwise (CCW) lightwave; the frequency difference between the driving frequencies of the two AOMs is equivalent to the Sagnac frequency difference caused by gyro rotation. Thus, the gyro output is observed. () 2006 Society of Photo-Optical Instrumentation Engineers.
\end{abstract}

[DOI: $10.1117 / 1.2280645]$

Subject terms: gyro; ring resonator; silica waveguide; phase modulation spectroscopy technology.

Paper 050997LRR received Dec. 21, 2005; revised manuscript received May 23, 2006; accepted for publication May 28, 2006; published online Aug. 25, 2006.

A small, robust, tactical-grade performance gyro is vital for the successful implementation of the smart weapons and surveillance apparatus. A waveguide-type optical passive ring resonator gyro (OPRG) is a promising candidate. ${ }^{1,2}$ The OPRG is a frequency-sensitive device. The gyro rotation is determined by the resonance frequency difference in the clockwise $(\mathrm{CW})$ and counterclockwise (CCW) lightwaves due to the Sagnac effect. ${ }^{1}$ Light circulating many turns in the ring resonator can build up the Sagnac effect. Thus, a short-length sensing loop is required. In this letter, the phase modulation (PM) spectroscopy technique is chosen to detect the gyro signal using $\mathrm{LiNbO}_{3}$ phase modulators. ${ }^{3}$ In the PM spectroscopy technique, the modulating signal and feedback signal do not interact, moreover the $\mathrm{LiNbO}_{3}$ phase modulators are easy to be integrated with other optical devices. They will make the OPRG compact. Figure 1 shows a schematic diagram of the experimental setup of the OPRG system. The resonator is composed of a 6-cm-long silica waveguide ring and a directional coupler (C4). The wavelength of the laser is $1550 \mathrm{~nm}$ and the diameter of the resonator is $1.9 \mathrm{~cm}$, so the scale-factor of the OPRG is ${ }^{1} 0.84 \times 10^{4} \mathrm{~Hz} / \mathrm{rad} \mathrm{s}^{-1}$.

0091-3286/2006/\$22.00 @ 2006 SPIE
The silica planar lightwave circuit (PLC) is the key rotating sensing element in the OPRG. It consists of two input/output directional couplers ( $\mathrm{C} 2$ and $\mathrm{C} 3$ ) and one resonator coupler $(\mathrm{C} 4)$. The coupling ratio of couplers $\mathrm{C} 2$ and $\mathrm{C} 3$ is designed as $50 \%$. The coupling ratio of coupler $\mathrm{C} 4$ is optimized by the total loss in the ring resonator, including propagation loss of silica waveguide ring, excess loss due to the curvature, and excess loss through coupler $\mathrm{C} 4$. Considering the temporal coherence of the laser, the resonance curve of a ring resonator is given by ${ }^{4,5}$

$R=\left(1-\alpha_{c}\right)\left[1-\rho \frac{(1-Q)^{2}}{(1-Q)^{2}+4 Q \sin ^{2}(\delta / 2)}\right]$

where $\rho$ is the resonance depth; $\delta$ is the phase shift around one trip of the ring; $Q=\left(1-\alpha_{R}\right)^{1 / 2}\left(1-k_{C}\right)^{1 / 2}(1$ $\left.-\alpha_{C}\right)^{1 / 2}[\exp (-\pi \Delta f \tau)] ; \alpha_{R}$ is the total propagation loss in the ring; $k_{C}$ and $\alpha_{C}$ are the coupling ratio and coupling loss of resonator coupler $\mathrm{C} 4$, respectively; $\Delta f$ is the spectral linewidth of the laser; and $\tau$ is the optical transmission time in the ring as $n L / c$. Here $n$ is the refractive index of the silica waveguide, $L$ is the ring length, and $c$ is the velocity of the light in the vacuum. Gyro sensitivity is maximized when the slope of resonance dip is the largest; namely, when the total loss in the ring resonator and the linewidth of the laser are determined, there is an optimum $k_{C}$ for gyro. Figure 2 shows the shot-noise-limited sensitivity and the finesse of the ring resonator versus the coupling ratio $k_{C}$. Here we use typical parameters of silica PLC and other devices. The losses of the waveguide and the coupler are assumed to be $0.01 \mathrm{~dB} / \mathrm{cm}$ and $0.1 \mathrm{~dB}$. The wavelength, spectral linewidth, and output power of the fiber laser are $1550 \mathrm{~nm}, 30 \mathrm{kHz}$, and $10 \mathrm{~mW}$, respectively. The quantum efficiency of the photodiode (PD) is 0.8 . The bandwidth of the measuring system is $1 \mathrm{~Hz}$. The coupling ratio of coupler C4 is variable.

In the experiments, the resonance curve of the silica waveguide ring resonator was tested first. A low-frequency sawtooth waveform at $1 \mathrm{~Hz}$ was applied to the fiber laser, and then the frequency of the fiber laser changed linearly with time. The output of the ring resonator was monitored by a PD. The oscilloscope trace of Fig. 3 shows the applied voltage and the resonance curve observed in the PD. In Fig. 3 , the applied voltage is a sawtooth wave with a voltage of about $20 \mathrm{~V}$ and a period of $1 \mathrm{~s}$. For a deeper resonance curve, the times corresponding to the free spectral range (FSR) and the full width at half maximum (FWHM) are $680 \mathrm{~ms}$ and $12.4 \mathrm{~ms}$, respectively. Because the frequency of the laser changes linearly with the input voltage at $250 \mathrm{MHz} / \mathrm{V}$, the frequency of the fiber laser changes linearly with time at $5000 \mathrm{MHz} / \mathrm{s}$. Thus, the FSR, FWHM, finesse $(F)$, and the resonance depth of this ring resonator are $3.4 \mathrm{GHz}, 62 \mathrm{MHz}, 54.8$, and $70 \%$, respectively. The shallower resonance dips caused by the unadjusted eigenstate of polarization in the resonator can also be seen in the upper curve in Fig. 3. This may be overcome by using two polarization controllers (PCs) to adjust the polarization state of the input light before the silica PLC, as shown in Fig. 1.

The oscilloscope trace of Fig. 4 shows the demodulation curves at the high frequency lock-in amplifiers (LIAs) for both $\mathrm{CW}$ and CCW lightwaves. The separation between 


\section{OE LETTERS}

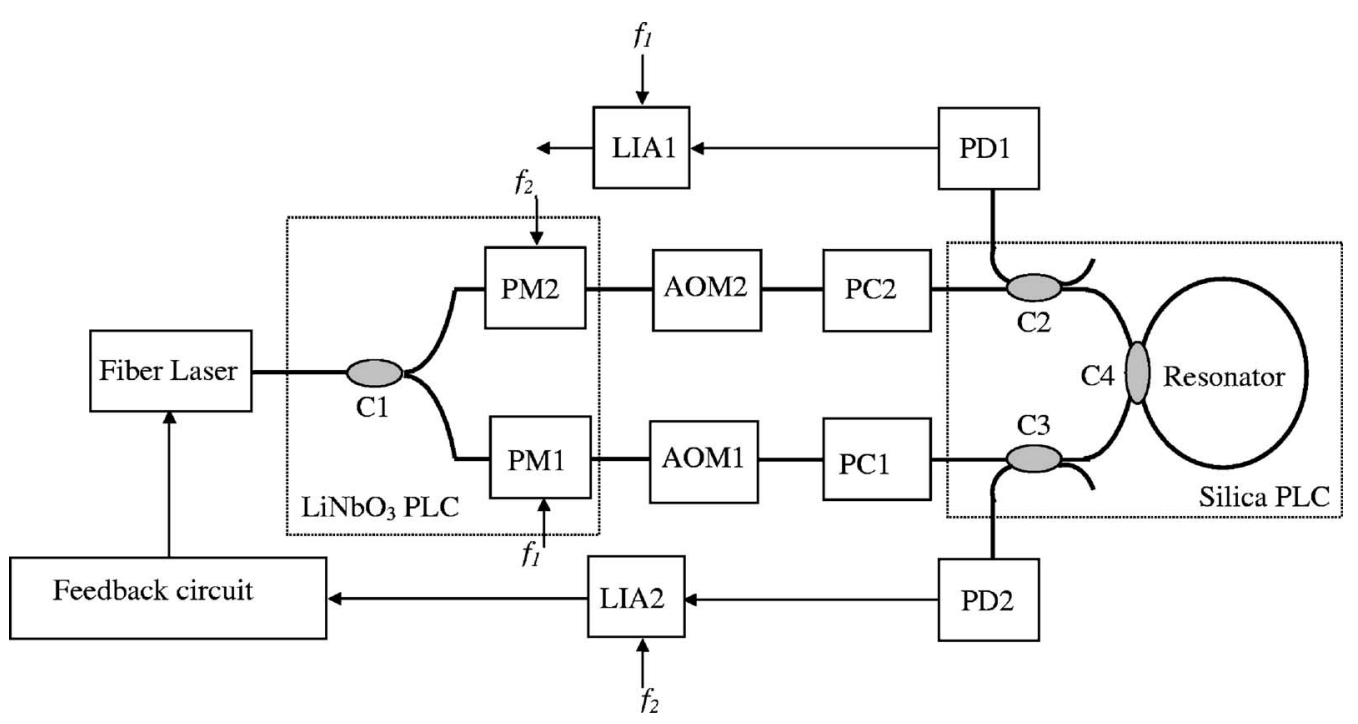

Fig. 1 Experimental setup of the OPRG using the PM spectroscopy technique.

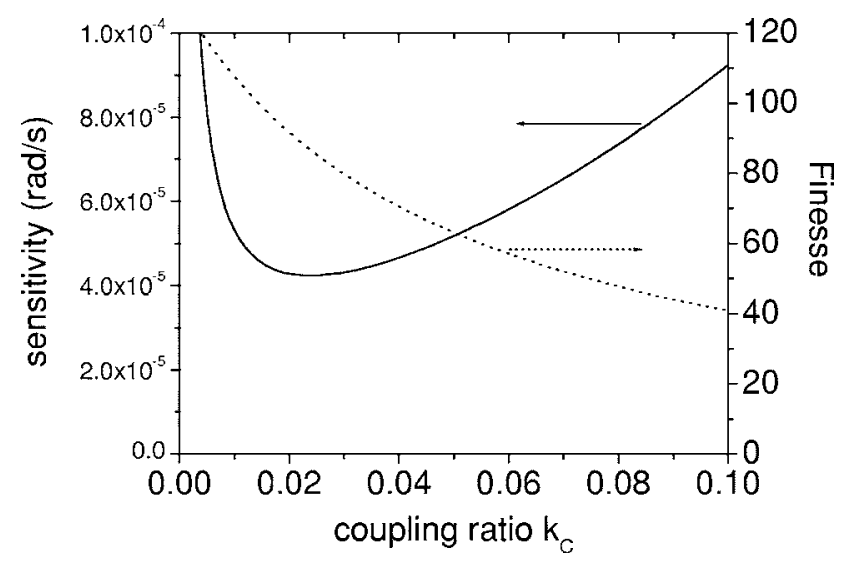

Fig. 2 Sensitivity and finesse versus coupling ratio $k_{C}$.

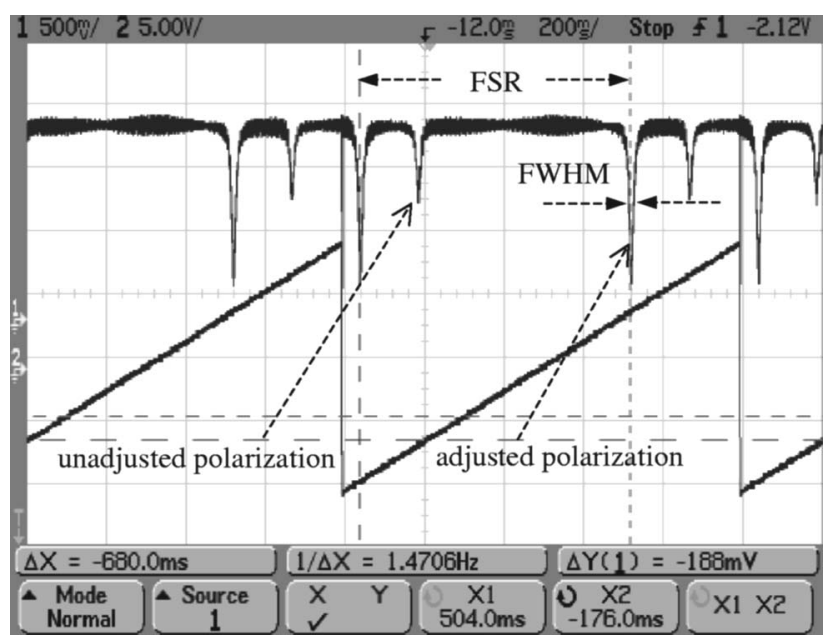

Fig. 3 Resonance curve of the fabricated silica PLC ring resonator.

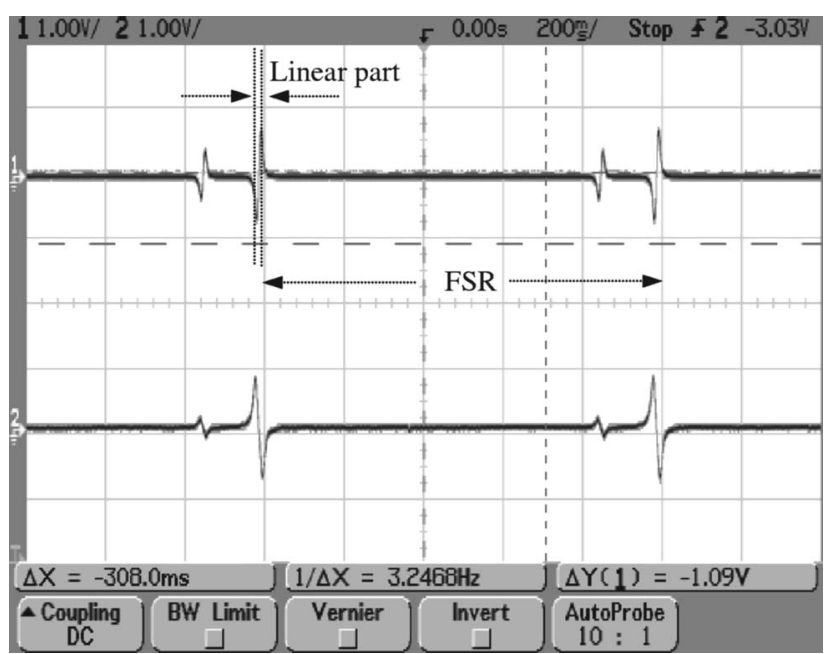

Fig. 4 Demodulation curves in CW and CCW directions.

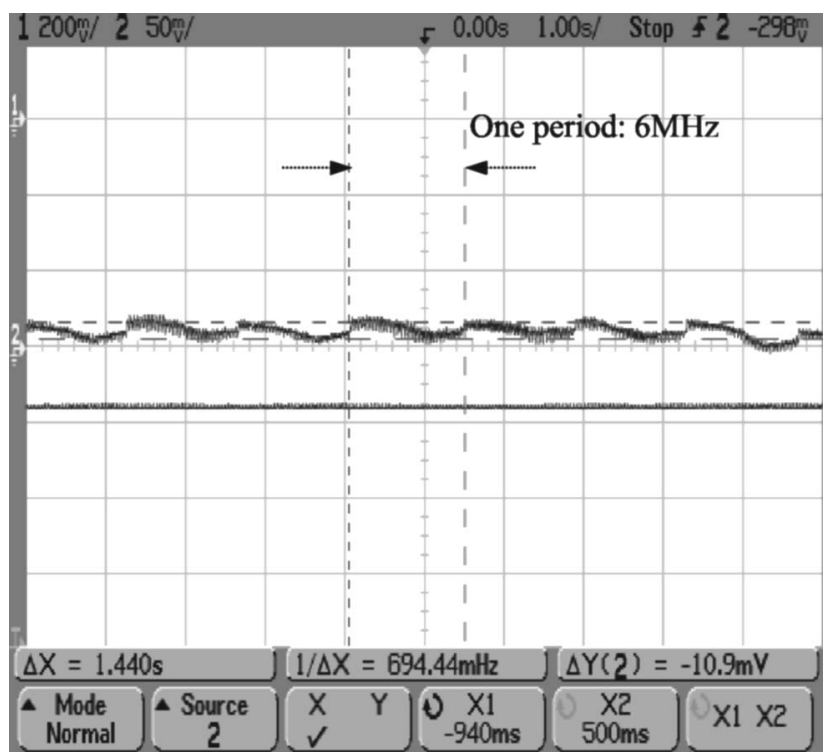

Fig. 5 Rotation observation with equivalent rotation input. 
each discriminant is the FSR. From Fig. 4, we notice that the demodulation curve has good linearity near the resonance point. The times corresponding to the linear part of the main resonance demodulation curve and one FSR are $26.7 \mathrm{~ms}$ and $1 \mathrm{~s}$, respectively. This means that the frequency deviation $\Delta f$ of the linear part is $90.78 \mathrm{MHz}$. The demodulation amplitude is $1.62 \mathrm{~V}$. Thus, the slope of the demodulation curve for linear part is $18 \mathrm{nV} / \mathrm{Hz}$, since the scale factor of this OPRG is $0.84 \times 10^{4} \mathrm{~Hz} / \mathrm{rad} \mathrm{s}^{-1}$. Considering the present level of the low noise detection circuit, a signal larger than $11 \mathrm{nV}$ can be measured. ${ }^{6}$ Thus, the detection sensitivity of this OPRG will be $7.3 \times 10^{-5} \mathrm{rad} / \mathrm{s}$. The discriminant corresponding to the shallower dips, as shown in Fig. 4, is also demodulated clearly. This verifies that the designed high-frequency LIA has high demodulation accuracy.

After having obtained the parameters of the resonator, the open-loop system was set up. The acousto-optical modulator AOM1 (AOM2) between the PM1 (PM2) and $\mathrm{C} 1$ (C2) is used to give additional frequency shift for the $\mathrm{CW}(\mathrm{CCW})$ lightwave. When the fiber laser frequency $f_{0}$ is locked to the resonance frequency $f_{\mathrm{CCW}}$ in the $\mathrm{CCW}$ lightwave by the feedback circuit, the PD output in the CCW lightwave is stable, as shown in the lower curve in Fig. 5. The difference between the driving frequencies of the two AOMs is equivalent to the Sagnac frequency difference due to gyro rotation. The driving frequency of AOM2 is $40 \mathrm{MHz}$, while the driving frequency of AOM1 changes linearly with time from 43 to $37 \mathrm{MHz}$. Thus, the equivalent Sagnac frequency difference between CCW and CW lightwaves will also change from 3 to $-3 \mathrm{MHz}$ linearly with time. The equivalent gyro output is shown in the upper curve in Fig. 5. As seen from Fig. 5, the gyro output corresponding to a $6-\mathrm{MHz}$ frequency difference is $10.9 \mathrm{mV}$. It is close to the calculated results $10.8 \mathrm{mV}$ from Fig. 4.

In this letter, an open-loop-operation OPRG based on the phase modulation spectroscopy technique was setup. The resonator parameters were measured and the detection sensitivity of this OPRG will be $7.3 \times 10^{-5} \mathrm{rad} / \mathrm{s}$. Additionally, the gyro output was observed with equivalent rotation input.

\section{Acknowledgments}

The authors would like to acknowledge the Excellent Young Teachers Program of MOE, China, for their support (2003 No. 355).

\section{References}

1. S. Ezekiel and R. Balsamo, "Passive ring resonator gyroscope," Appl. Phys. Lett. 30(9), 478-480 (1977).

2. K. Suzuki, K. Takiguchi, and K. Hotate, "Monolithically integrated resonator microoptic gyro on silica planar lightwave circuit," $J$. Lightwave Technol. 18(1), 66-72 (2000).

3. X. Zhang, H. Ma, C. Ding, and Z. Jin, "Analysis on phase modulation spectroscopy of resonator fiber optic gyro," Chin. J. Lasers 32(11) 1529-1533 (2005)

4. H. Ma, Z. Jin, C. Ding, and Y. Wang, "Influence of spectral linewidth of laser on resonance characteristics in fiber ring resonator," Chin. J. Lasers 30(8), 731-734 (2003).

5. H. Ma, Z. Jin, C. Ding, and Y. Wang, "Research on signal detection method of resonator fiber optical gyro," Chin. J. Lasers 31(8), 10011005 (2004).

6. G. Yang, H. Ma, X. Zhang, K. Zhou, and Z. Jin, "Design of high frequency lock-in amplifier applied in R-MOG," Chin. J. Sens. Actuat. 18(4), 863-866 (2005). 